T. Kawamoto

Nagoya Math. J.

Vol. 143 (1996), 13-29

\title{
THE CONVERGENCY OF SOME ROUTING PROTOCOLS
}

\author{
TAKUJI KAWAMOTO
}

\section{Introduction}

\subsection{Conventional routings}

In every network, many nodes with brains are connected by many links each other. Some nodes are, for example, computers, terminals, or routers. The both ends of a link can communicate directly each other. For example, local area network, in which every nodes are directly connected each other, looks like Figure 1 , then every nodes can communicate directly to others.

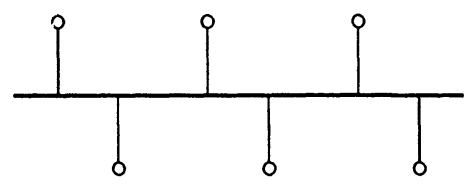

FIGURE 1. Local area network.

But, in global area network, it is difficult to connect every nodes directly, so they have to communicate through intermediate nodes. In this case, we encounter a routing problem, that is, there may exist many routes to the destination, if the network has reticulated links as Figure 2.

Up to now, every routes were not so complicated. Therefore, we took a way that persons give a certain route to the destination in advance or routing information protocol (RIP) (Hedrick [5] and Mills [8]), and for wide area network, took the method called core gateway system or autonomous system.

We explain some algorithms first for complicated routes.

The RIP is a protocol in a series of routing protocols based on the BellmanFord (or distance vector) algorithm (Ford and Fulkerson [4]).

Each nodes decide the shortest route to the destination by the RIP

Received February 23, 1993.

Revised May 22, 1995. 
communication between neighbouring nodes, that is, nodes decide dynamically the hop count number which is the smallest number of the entirely relay nodes in a route, which runs through all of the routes to a given destination.

In other words, each node announce a hop count number that is the smallest number, which neighbour nodes announced last time, plus 1 .

But, this protocol has a problem called "slow convergence" or "count to infinity" (Theorem 3.2).

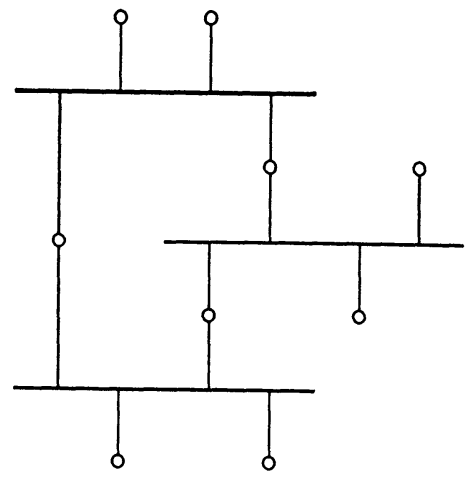

FIGURE 2. Wide area network.

There have been proposed some improved methods of RIP which avoid this problem. One is by "assumption a certain distance as unreachable", and the second is "split horizon update", and is "hold down", and the last is "poison reverse with triggered updates".

"assumption a certain distance as unreachable" is a method considering the destination to be unreachable when the number of it's hop count exceeds a threshold which is determined in advance. So there is the upper bound of the length of the links.

We do not describe detail for "split horizon update", but in this method, routing does not function so effectively, when its topology has some long loops.

Moreover, since RIP can not correspond dynamically to the crowdness of network, these routing controls are static one.

Therefore, in order to correspond to a dynamic change of the network load, the HELLO routing protocol (Mills [11]) were suggested.

This protocol is as like as RIP but delay between nodes instead of hop count.

These typical protocols are introduced in Comer [3] and variations are treated in Bertsekas [2].

But there is no essential solution of the problem "slow convergence", so this 
method is not intended for practical use, because the method of "assumption a certain distance as unreachable" restricts the number of nodes joined.

The last of all, the methods called core gateway system and called autonomous system is suggested. Internet has been grown adding new networks to ARPANET. Core gateway system is used to add these sub networks. Each sub network is linked to ARPANET at core gateway, which has informations for that sub network. Core gateways destermine route to destination network by talking each other. If there is a packet which we want to send to the host $\mathrm{H}$ in the network $\mathrm{N}$, that packet is sent to the core gateway which connects to $\mathrm{N}$ first, and is sent to $\mathrm{H}$ in $\mathrm{N}$ next.

Autonomous system is a developed one of core gateway system.

In autonomous system, we divide the whole of network to some sub autonomous systems, and we restrict routing controls to each sub system and to coset of sub systems, and each sub system may contain sub sub systems.

In these methods, the control of each system is left each organization, and the routing protocol is also various, that is the method by hand, RIP, HELLO, or etc.

Since Internet is the aggregation of many networks which are owned each organizations, these method is useful, but since each of sub systems is linked by only one link, we have some faults, those are the increase of traffic at there, and the weakness for breaking line. So these methods are useless in order to expand to wide complicated network.

So, in order to avoid the problem "slow convergence", we will define another method, modelize it, and prove mathematically that the "decision of arrival" is warranted in arbitrary network without restriction of it's topology.

\subsection{Suggestion of new routing}

This new protocol is based on the RIP and the HELLO protocol. Each nodes have a common period ${ }^{1}$ and they are synchronized.

In the HELLO protocol, for the given destination node $w$, each node which wants to decide the route to $w$ renews the delay database to $w$ every period.

Each node communicates the information of the delay database at current period, and determine the value of database at the next period.

The method to determine it is the way that the delay database at each node at the current period is calculated as follows, first, for all neighbourhood nodes, consider additions of the delay databases at the last period to the delay times between the watching node and its neighbourhoods, and select the least value among these values for the delay database.

\footnotetext{
1 In the actual protocol, more complicated synchronous method is taken.
} 
Now, in order to avoid "slow convergence", we provide the following assumption,

Assumption. Consider the value of a delay database renewed at each node every period. It must be constant, decreasing, or infinite, and the information infinite has to be told as "emergency information" to neighborhoods in an instant.

The exact definition is described in Section 2.1.

Then, we must show that the convergency problem is solved in such given routing protocol, that is as follows.

QUESTION 1.1. Consider a routing protocol which is given as previous. If a crowdness converges, does the routing which decided by that algorithm converge?

This paper's principal purpose is to give a solution to this problem.

\subsection{Outline}

First of all, in Section 2.1, we modelize the routing protocols (Definition 2.9), and moreover, define delay function and it's convergency. The convergency is an important property. In this section, we use basic terms of the ordinal numbers, which are introduced in Jech [6] or Kenneth [7].

Then we define some routing models, such as RIP (Example 2.8), the HELLO routing protocol (Example 2.7), and decreasing routing protocol (Definition 2.10) which we suggest.

Next, in Section 2.2, we consider lower dependency (Definition 2.13) and prove convergency of delay functions (Theorem 2.3).

In Section 2.3, we discuss the relation of convergency and connectivity (Theorems 2.6 and 2.8). Then we prove equivalency of convergency and connectivity when routing protocol is HELLO or the decreasing routing protocol (Corollary 2.10).

Finally, in the last section (Section 3), we prove the convergency of RIP (Theorem 3.1) and the decreasing routing protocol (Theorem 3.3), and show an example that HELLO does not always converge (Theorem 3.2). 


\section{A modelization of routings}

\subsection{The definition of routing}

Definition 2.1. Let $T$ be an ordinal number, which has no maximal elements. We call it a time set.

Remark. Definition 2.1 is equivalent to that $T$ is a limit number, and that the upper limit of any subset in $T$ is in it.

Example 2.1. $T=\omega=\{0,1,2, \ldots\}$ is a time set. It is called the descrete time.

Definition 2.2. Let $V$ be an ordinal number with the maximal element $\infty$, which forms a semi group by (not necessary commutative) addition, and satisfies the followings,

(1) $h+\infty=\infty+h=\infty$ for each $h \in V$,

(2) the minimum element 0 of $V$ is the zero element, that is, $h+0=0+h$ $=h$ for each $h \in V$,

(3) $h+h^{\prime}>h$ for each $h(\neq \infty), h^{\prime}(\neq 0) \in V$.

We call it a hop value set.

EXAmple 2.2 (natural ordinal numbers). Let $\alpha$ be an ordinal number, and denote $\dot{+}$ its natural addition, and let $V=\alpha \dot{+} 1=\alpha \cup\{\infty=\alpha\}$ further. If we define an addition + by the followings,

$$
h+h^{\prime}=\left\{\begin{array}{cc}
\infty, & h \dot{+} h^{\prime} \geq \infty, \\
h+h^{\prime}, & h \dot{+} h^{\prime}<\infty,
\end{array}\right.
$$

then $V$ is a hop value set.

EXAmPLE 2.3 (another example of hop value set). Let $\alpha$ be an ordinal number, and denote $\dot{x}$ its natural multiplication. Let $V=(\alpha \dot{+} 1) \backslash\{0\}=\alpha \backslash\{0\} \cup\{\infty$ $=\alpha$ f further. If we define an addition + by the followings

$$
h+h^{\prime}= \begin{cases}\infty, & h \dot{\times} h^{\prime} \geq \infty, \\ h \dot{\times} h^{\prime}, & h \dot{\times} h^{\prime}<\infty,\end{cases}
$$

then $V$ is a hop value set. 
Example 2.4 (natural numbers). In the definition of Example 2.2, let $\alpha=\omega$, then $\alpha$ is a hop value set.

EXAmple 2.5 (finite sets). Fix an integer $n>0$, and assume $\alpha=n=\{0,1, \ldots$, $n-1\}$ further in Example 2.1. Then it is a hop value set.

Definition 2.3. We call $G=\{\mathscr{V}, \mathscr{E}\}$ a graph, when $\mathscr{V}$ is finite, $\mathscr{E}$ is a subset in $\mathscr{V} \times \mathscr{V}$, and they satisfy the followings,

(1) $(v, w) \in \mathscr{E} \Rightarrow(w, v) \in \mathscr{E}$ for each $v, w \in \mathscr{V}$,

(2) $v \in \mathscr{V} \Rightarrow(v, v) \notin \mathscr{E}$.

We call $\mathscr{V}$ a verticies set of $G$, and $\mathscr{E}$ an edges set of $G$.

Remark. We regard for convenience' sake the set of edges $\mathscr{E}$ contains no diagonal elements.

EXAmPLE 2.6 (networks). A Network is considerd a graph, by regarding $\mathcal{N}$ as the whole of nodes, and $\mathscr{L}$ as the set of these links.

Definition 2.4. Let $G=\{\mathscr{V}, \mathscr{E}\}$ be a graph. We denote $N(v)$ and $C_{\infty}(v)$ respectively the neighbourhood of $v$ and the connected component of $v$. These are defined as follows,

$$
\begin{aligned}
& C_{0}(v)=\{v\}, \\
& C_{n}(v)=\left\{\left.w \in \mathscr{V}\right|^{\exists} w^{\prime} \in C_{n-1}(v),\left(w^{\prime}, w\right) \in \mathscr{E}\right\} \cup C_{n-1}(v), \\
& N(v)=C_{1}(v) \backslash C_{0}(v), \\
& C_{\infty}(v)=\lim _{n \rightarrow \infty} C_{n}(v) .
\end{aligned}
$$

You see $C_{n}(v) \supset C_{n-1}(v)$ clearly, and the limit in the formula (2.4) terminates at some $n$.

Definition 2.5. We fix a time set $T$, a hop value set $V$ and a graph $G=$ $\{\mathscr{V}, \mathscr{E}\}$. We call a function $\varphi: T \times \mathscr{E} \rightarrow \backslash\{0\}$ the crowdness function. We denote $\mathscr{C}_{G}$ the whole crowdness functions on a graph $G$.

Definition 2.6. We say that an edge $e$ is broken at a time $t$, when a crowdness $\varphi(t, e)$ equals infty $\infty$, and that a crowdness function $\varphi$ converges, when there exists some time $t \in T$ such that $\varphi\left(t_{1}, e\right)=\varphi\left(t_{2}, e\right)$ holds for any $t_{1}$, 
$t_{2}(\geq t) \in T, e \in \mathscr{E}$

Definition 2.7. Let $G=\{\mathscr{V}, \mathscr{E}\}$ be a graph, and define another graph $G^{\prime}=$ $\left\{\mathscr{V}^{\prime}, \mathscr{E}^{\prime}\right\}$ with $\mathscr{V}^{\prime}=\mathscr{V}$ and $\mathscr{E}^{\prime}=\{e \in \mathscr{E} \mid \varphi(t, e)$ converges to a finite value $\}$ where $\varphi$ is a given convergent crowdness function, and $N_{\varphi}(v), C_{\varphi, 1}(v)$ and $C_{\varphi, \infty}(v)$ be sets as same as defined by $G^{\prime}$ but no symbol $\varphi$ respectively.

Definition 2.8 (routings). Let a time set $T$ and a hop value set $V$ be fixed.

Routing is a function $\rho: T \times V \times 2^{V} \rightarrow V$. We denote $\mathscr{R}$ the whole routing functions set.

Definition 2.9 (routing protocols (based on the Bellman-Ford algorithm)). Routing protocol is a quintuple $\{T, V, G, C, \rho\}$ where a time set $T$, a hop value set $V$, a graph $G=\{\mathscr{V}, \mathscr{E}\}$, a set of crowdness functions $C \subset \mathscr{C}_{G}$, and a routing $\rho \in \mathscr{R}$.

Example 2.7 (the hello routing protocol (Mills [11])). We fix a time set $T=$ $\omega$ and a hop value set $V=\omega+1$ and let $G=\{\mathscr{V}, \mathscr{E}\}$ be an arbitrary graph.

Let $C=\mathscr{C}_{G}$ be a set of crowdness functions, and define a routing $\rho \in \mathscr{R}$ as

$$
\rho(t, h, H)=\rho_{\min }(t, h, H)=\min H,
$$

for each $t \in T, h \in V, H \in 2^{V}$. We call $\left\{\omega, \omega \dot{+} 1, G, \mathscr{C}_{G}, \rho_{\min }\right\}$ the Hello routing protocol.

EXAMPLE 2.8 (the routing information protocol (RIP) (Hedrick [5], Malkin [8]). Pick a suitable integer $n$, that is ordinary 16 , and fix a time set $T=\omega$ and a hop value set $V=n+1=\{0,1, \ldots, n-1, n=\infty\}$, and leave $G=\{\mathscr{V}, \mathscr{E}\}$ ordinal ly graph.

The $R I P$ is the quintuple $\left\{\omega, F_{n}, G, \mathscr{C}_{G}^{\mathrm{RIP}}, \rho_{\mathrm{min}}\right\}$, where the set of crowdness functions $C \subset \mathscr{C}_{G}$ is defined by the followings,

$$
C=\mathscr{C}_{G}^{\mathrm{RIP}}=\{\varphi \in \mathscr{C} \mid \#\{\varphi(t, e)<\infty \mid t \in T\} \leq 1 \text { for every } e \in \mathscr{E}\},
$$

and the routing by $\rho=\rho_{\min }$.

Definition 2.10 (a decreasing routing protocol with triggered disconnection). Let a time set $T=\omega$, hop value set $V=\omega+1$ and $t_{p}(>1) \in T$ be fixed, and $G=\{\mathscr{V}, \mathscr{E}\}$ be an arbitrary set.

Consider the quintuple $\left\{\omega, \omega+1, G, \mathscr{C}_{G / t_{p}}, \rho_{\downarrow}\right\}$, where the crowdness set is 
$C=\mathscr{C}_{G / t_{p}}=\left\{\varphi \in \mathscr{C}_{G} \mid \varphi(t, e)=\varphi\left(t_{p}\left\lfloor t / t_{p}\right\rfloor, e\right)\right\}$ and the routing is defined as follows,

$$
\begin{aligned}
& \text { For each } t \in T, h \in V, H \in 2^{V} \\
& \rho(t, h, H)=\rho_{\downarrow}(t, h, H) \\
& = \begin{cases}\min H, & \min H \leq h \text { and } t \text { divides } t_{p}, \\
h, & \min H \leq h \text { and } t \text { does not divide } t_{p}, \\
\infty, & \min H>h .\end{cases}
\end{aligned}
$$

We call it decreasing routing protocol with triggered disconnection or decreasing routing protocol briefly.

Definition 2.11. Let $\{T, V, G=\{\mathscr{V}, \mathscr{E}\}, C, \rho\}$ be an arbitrary routing protocol. The delay function

$$
H_{\varphi, \rho}: T \times \mathscr{V} \times \mathscr{V} \rightarrow V
$$

is defined inductively for a crowdness function $\varphi \in C$ and a routing $\rho$, as follows,

(1) $H_{\varphi, \rho}(t, v, v)=0$ for each $t \in T$ and $v \in \mathcal{V}$,

(2) For each $t \in T$ and $v, w \in \mathscr{V}(v \neq w)$,

(a) in case of $t=0$, we put $H_{\varphi, \rho}(0, v, w)=\infty$,

(b) in case that $t$ is a succesor,

using the set of the hop values

$$
W=\left\{H_{\varphi, \rho}(t \dot{-} 1, x, w)+\varphi(t \dot{-} 1,(v, x))\right\}_{x \in N(v)} \cup\{\infty\},
$$

we define

$$
H_{\varphi, \rho}(t, v, w)=\rho\left(t, H_{\varphi, \rho}(t-1, v, w), W\right),
$$

(c) in case that $t$ is a limit number,

we set

$$
H_{\varphi, \rho}(t, v, w)=\varlimsup_{\lim _{s \rightarrow t} H_{\varphi, \rho}(s, v, w) .}
$$

$A$ delay function with a destination $w$ is a $\operatorname{map} H_{\varphi, \rho}(\cdot, \cdot, w): T \times \mathscr{V} \rightarrow V$.

Remark. We note that routing is, the other way, a kind of the calculation of delay function. That is, if we obtain the delay function's value on a point and on around points, it calcules the next step value of that function on that point.

We note furthermore that its culculation is independent to given point, and the crowdness function is considered the weight of the edges. 
Definition 2.12. Let $H_{\varphi, \rho}$ be a delay function with destination $w \in \mathscr{V}$. We say that $H_{\varphi, \rho}$ converges at $v \in \mathscr{V}$ when there exists some time $t \in T$ such that $H_{\varphi, \rho}\left(t_{1}, v, w\right)=H_{\varphi, \rho}\left(t_{2}, v, w\right)$ for each $t_{1}, t_{2}(\geq t) \in T$.

We say that $H_{\varphi, \rho}$ diverges at $v \in \mathscr{V}$ when there exists some time $t \in T$ for each hop value $h \in V$ except $\infty$, such that $H_{\varphi, \rho}(t, v, w)>h$.

Lemma 2.1. The definition of a delay function $H_{\varphi, \rho}$ is independent of both the crowdness function $\varphi$ and the routing $\rho$.

Proof. If you read Definition 2.11 carefully, you will find that it is defined by the transfinite induction by the time set. So its uniqness is evidently.

\subsection{The convergency}

The following definition seems to be a natural condition.

Definition 2.13. We say the routing $\rho$ is lower dependent, if the following statement is satisfied.

For three tupples $t_{1}, t_{2} \in T, h_{1}, h_{2} \in V, H_{1}, H_{2} \in 2^{V}$, we may assume that $r_{i}=\rho\left(t_{i}, h_{i}, H_{i}\right)(i=1,2)$ and $r=r_{1} \leq r_{2}$ because of these symmetry, then

$$
h_{1} \geq r, h_{2} \geq r \text { and } H_{1} \cap[0, r]=H_{2} \cap[0, r] \Rightarrow r_{1}=r_{2} \text { or } r_{2}=h_{2} \text {. }
$$

Remark. You may think the conclusion $r_{2}=h_{2}$ in the Definition 2.13 is very strange, but it becomes finally to the equation $r_{2}=r_{1}$, in the proof of Theorem 2.3 .

But since the description of Definition 2.13 is more suitable than a definition without the conclusion $r_{2}=h_{2}$, We dare to describe by this form.

Lemma 2.2. The all routing protocols mentioned in Examples 2.7, 2.8 and Definition 2.10 are lower dependent.

Proof. Each definitions make us understood easily.

Theorem 2.3. Let $\{T, V, G=\{\mathscr{V}, \mathscr{E}\}, C, \rho\}$ be an arbitrary routing protocol, and furthermore $\varphi$ is convergent, $\rho$ is lower dependent. Then there exists some limit number $\mu \in V$ and some stair function $h<h^{\prime} \Rightarrow f(h)<f\left(h^{\prime}\right)$, such that

$$
{ }^{\exists} t \geq f(h), H_{\varphi, \rho}(t, v, w)=h \Rightarrow{ }^{\forall} t \geq f(h), H_{\varphi, \rho}(t, v, w)=h,
$$


for each $v, \omega \in \mathscr{V}$ and $h \in[0, \mu)$. If the time set $T$ is bigger enough, $\mu$ can be chosen larger.

Proof. We prove formula (2.6) by transfinite induction by $h$. However, we assume it as we like that the time set is bigger enough if $h$ is a limit number.

(1) In the case of $h=0$.

We choose $f(0)$ for the condition

$$
\varphi\left(t^{\prime}, e\right)=\varphi\left(t^{\prime \prime}, e\right)
$$

is concluded for each $t^{\prime}, t^{\prime \prime}(\geq f(0)) \in T, e \in \mathscr{E}$. The following condition make us find that formula (2.6) is correct,

$$
\begin{aligned}
{ }^{\exists} t \in T, 0=H_{\varphi, \rho}(t, v, w) & \Leftrightarrow v=w \\
& \Leftrightarrow{ }^{\forall} t \in T, 0=H_{\varphi, \rho}(t, v, w) .
\end{aligned}
$$

(2) In the case of $h \neq 0$ and formula (2.6) is correct for the values which are less than $f$.

Let $\hat{f}(h) \in T$ be $\lim _{g \uparrow h} f(g)=\inf \{f(g) \mid g<h\}$ if $h=g+1$ is a limit number, and be $f(g)$ otherwise. An impossibility that $T$ contains the value $\lim _{g \uparrow h} f(g)$ may be considerd, but we can avoid it by taking $T$ big-

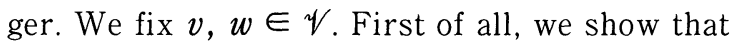

$$
H_{\varphi, \rho}(t, v, w)=h \Rightarrow H_{\varphi, \rho}(t+1, v, w) \leq h
$$

for each $t>\hat{f}(h)$.

Now, we find $n=H_{\varphi, \rho}(t-1, v, w) \geq h$, in fact, if we assume $n<h$ namely $n \leq g$, then we have $t \dot{-} \geq \hat{f}(h) \geq f(n)$. Therefore by the assumption of induction we obtain $n=H_{\varphi, \rho}(t, v, w)=h$. These contradict $n<h$.

Next, we put

$$
\begin{aligned}
& t_{1}=t \\
& h_{1}=H_{\varphi, \rho}(t-1, v, w)=n \geq h \\
& H_{1}=\left\{H_{\varphi, \rho}(t-1, x, w)+\varphi(t-1,(v, x))\right\}_{x \in N(v)} \cup\{\infty\} \\
& r_{1}=\rho\left(t_{1}, h_{1}, H_{1}\right) \\
& t_{2}=t+1 \\
& h_{2}=H_{\varphi, \rho}(t, v, w)=h \\
& H_{2}=\left\{H_{\varphi, \rho}(t, x, w)+\varphi(t,(v, x))\right\}_{x \in N(v)} \cup\{\infty\} \\
& r_{2}=\rho\left(t_{2}, h_{2}, H_{2}\right)
\end{aligned}
$$


then

$$
\begin{aligned}
r_{1} & =\rho\left(t_{1}, h_{1}, H_{1}\right) \\
& =\rho\left(t, H_{\varphi, \rho}(t-1, v, w), H_{1}\right) \\
& =H_{\varphi, \rho}(t, v, w) \\
& =h \\
r_{2} & =\rho\left(t_{2}, h_{2}, H_{2}\right) \\
& =\rho\left(t+1, H_{\varphi, \rho}(t, v, w), H_{2}\right) \\
& =H_{\varphi, \rho}(t+1, v, w)
\end{aligned}
$$

is obtained by the definition of $H_{\varphi, \rho}$. We shall derive contradiction by assuming $h<r_{2}$ which is the negation of the conclusion $h \geq r_{2}$.

We shall check the assumption of the Definition 2.13. We find $r=r_{1}=$ $h<r_{2}$ and obtain

$$
\begin{aligned}
& H_{1} \cap[0, r]=H_{1} \cap[0, n] \\
& =\left\{h=H_{\varphi, \rho}(t-1, x, w)+\varphi(t \dot{-} 1,(v, x)) \mid h \leq n\right\}_{x \in N(v)} \cup\{\infty\} \\
& \begin{aligned}
H_{2} \cap[0, r] & =H_{2} \cap[0, n] \\
& =\left\{h=H_{\varphi, \rho}(t, x, w)+\varphi(t,(v, x)) \mid h \leq n\right\}_{x \in N(v)} \cup\{\infty\} .
\end{aligned}
\end{aligned}
$$

Therefore $H_{\varphi, \rho}$ 's are all less than $h$, and $t \dot{-} 1 \geq \hat{f}(h) \geq f\left(H_{\varphi, \rho}\right)$ is hold. If we use the assumption of induction, then we have

$$
H_{\varphi, \rho}(t \dot{-} 1, x, w)=H_{\varphi, \rho}(t, x, w) .
$$

Furthermore $H_{1} \cap[0, r]=H_{2} \cap[0, r]$ is obtained, because the condition (2.7) leads $\varphi(t \dot{-} 1,(v, x))=\varphi(t, v, x))$.

We get $h_{1} \geq r, h_{2} \geq r$ easily too, we find the assumption of the Definition 2.13 is completely satisfied. So that we get finally $r_{1}=r_{2}$ or $r_{2}=$ $h_{2}$. These contradict $r_{2}>r_{1}=h=h_{2}$.

Now we get correctness of formula (2.8). then we shall examine for more detail. Formula (2.8) says that if $H_{\varphi, \rho}$ takes the value $h$ once on the time $t$ $>\hat{f}(h)$, then it holds $h$ otherwise it becomes less than $h$, and that if it takes the value less than $h$, then it never decreases, because of the assumption of induction. So, we obtain the following three situations, there exists $\tilde{f}(h)<s_{1} \leq s_{2} \leq T$ such that

$$
H_{\varphi, \rho}(t, v, w) \begin{cases}>h, & \hat{f}(h)<t<s_{1}, \\ =h, & s_{1} \leq t \leq s_{2}, \\ =h^{\prime}(<h), & s_{2} \leq t\end{cases}
$$


Namely, we get finally the other three situations,

(a) for each $t \geq \hat{f}(h)+1, H_{\varphi, \rho}(t, v, w)>h$,

(b) there exists $\hat{f}(h)<s$ such that $H_{\varphi, \rho}(t, v, w)=h$ for each $t \geq s$,

(c) there exists $\hat{f}(h)<s$ such that $H_{\varphi, \rho}(t, v, w)<h$ for each $t \geq s$.

Now we put $f(h)$ the largest one among previous $\hat{f}(h) \dot{+} 1$ and $s$ 's for all $v, w \in \mathcal{V}$, then for each situations, we obtain the last three situations,

(a) for each $t \geq f(h), H_{\varphi, \rho}(t, v, w)>h$,

(b) for each $t \geq f(h), H_{\varphi, \rho}(t, v, w)=h$,

(c) for each $t \geq f(h), H_{\varphi, \rho}(t, v, w)<h$.

So we get the conclusion formula (2.6) of induction.

COROLLARY 2.4. If we suppose the condition of Theorem 2.3 and additionally we can take $\mu=\infty$, that is in Example 2.4 or $T$ is big enough, $H_{\varphi, \rho}(\cdot, v, w)$ converges or deverges for each $v, w \in \mathscr{V}$.

Proof. This corollary follows from Theorem 2.3.

COROLLARY 2.5. Under the condition of Theorem 2.3, if the hop value set $V$ is a finite set, $H_{\varphi, \rho}(\cdot, v, w)$ converges any value including $\infty$, for each $v, w \in \mathscr{V}$.

Proof. This corollary follows from Theorem 2.3.

\subsection{Connected components of the destination $w$}

In this section, we fix $T, V, \rho$, and let $G, C$ be arbitrary graph and crowdness function, where $\{T, V, G=\{\mathscr{V}, \mathscr{E}\}, C, \rho\}$ is a given routing protocol, and we assume the situation in Theorem 2.3 and $\mu=\infty$. Furthermore, we discuss with fixing the destination $w$.

Remark. The equation $\mu=\infty$ is independent of neither crowdness functions nor graphs.

Corollary 2.4 says that $H_{\varphi, \rho}(\cdot, v, w)$ will converge or divege, if we decide the destination $w$ and the vertex $v$ which we notice.

Then we divide $\mathscr{V}$ into the following two subsets,

$$
\mathscr{V}=\mathscr{V}_{<\infty} \cup \mathscr{V}_{\uparrow \infty},
$$

where

$$
\mathscr{V}_{<\infty}=\left\{v \in \mathscr{V} \mid H_{\varphi, \rho}(\cdot, v, w) \text { converges }\right\}
$$




$$
\mathscr{V}_{\uparrow \infty}=\left\{v \in \mathscr{V} \mid H_{\varphi, \rho}(\cdot, v, w): \text { divergent }\right\} .
$$

Furthermore we divide $\mathscr{V}$ into another two subsets,

$$
\mathscr{V}=\mathscr{V}_{-w} \cup \mathscr{V}_{+w}
$$

where

$$
\begin{aligned}
& \mathscr{V}_{-w}=C_{\varphi, \infty}(w) \\
& \mathscr{V}_{+w}=\mathscr{V} \backslash \mathscr{V}_{-w} .
\end{aligned}
$$

THEOREM 2.6. Under the previous conditions, consider the following two conditions.

(1) $\mathscr{V}_{<\infty} \subset \mathscr{V}_{-w}$, for cach $G, C$.

(2) For each $t \in T, h \in V, H \in 2^{V}$,

$$
\rho(t, h, H) \leq h \Rightarrow \rho(t, h, H) \geq \rho_{\min }(t, h, H) .
$$

Then condition (2) $\Rightarrow$ condition (1).

Proof. We assume condition (2). For arbitrary given $v \in \mathcal{V}_{<\infty}$, let $h$ be its convergent value, and we shall show $v \in \mathscr{V}_{-w}$ by transfinite induction by $h$.

First of all, in the case of $h=0$, the conclusion of induction is correct, because $h=0 \Leftrightarrow v=w$.

Next, we assume $h>0$.

Theorem 2.3 says that $H_{\varphi, \rho}(t, v, w)=h$ holds for each $t \geq f(h)$. It follows the definition of delay function that $H_{\varphi, \rho}(t+1, v, w)=\rho\left(t+1, H_{\varphi, \rho}(t, v, w), W\right)$ where $W \in 2^{V}$ is defined in formula (2.5).

That is $h=\rho(t+1, h, W)$, and with condition $(2)$, we will find that there exists $x \in N(v)$ such that $h \geq H_{\varphi, \rho}(t, x, w)+\varphi(t,(v, x)) . \varphi(t,(v, x))>0$ implies $h>h^{\prime}=H_{\varphi, \rho}(t, v, w)$. Here, Theorem 2.3 says also that $H_{\varphi, \rho}\left(t^{\prime}, x, w\right)=$ $h^{\prime}$ is satisfies for each $t \geq f\left(h^{\prime}\right)$. So, $x \in \mathscr{V}_{<\infty}$. The assumption of the induction drives $x \in \mathscr{V}_{-w}$, and $v \in \mathscr{V}_{-w}$ follows.

LEMma 2.7. Routing protocols specified in Example 2.7 and Definition 2.10 both satisfies the assumption of Theorem 2.6 and condition (2).

Proof. Easy to check.

THEOREM 2.8. Assume that we have $h, h^{\prime}(<\infty) \in V \Rightarrow h+h^{\prime}<\infty$ in addi. 
tion. Consider the following two conditions.

(1) $\mathscr{V}_{\uparrow \infty} \subset \mathscr{V}_{x w}$ for each $G, C$.

(2) For each $t \in T, h \in V, H \in 2^{V}$ there exists $t^{\prime}(>t) \in T$ such that $\rho\left(t^{\prime}, h^{\prime}, H^{\prime}\right) \leq \rho_{\min }\left(t^{\prime}, h^{\prime}, H^{\prime}\right)$ for each $h^{\prime} \in V, H^{\prime} \in 2^{V}$.

Then condition $(2) \Rightarrow$ condition (1).

Proof. We assume condition (2). If necessary, we consider large time enough, we can assume $\varphi$ is a constant. Its concrete value is $f(0)$ that is shown in the proof of Theorem 2.3. Furthermore, we consider larger time, we can also assume each delay functions on $\mathscr{V}_{<\infty}$ are constant. Its concrete value is also specified in

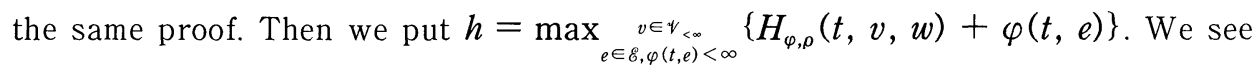
that its right-hand expression is a finite set, so we find that the maximum value of its right-hand expression exists and $h<\infty$ holds.

We can replace a time of large one which is more than $f(h+1)$ where $f$ is given in Theorem 2.3 .

Now, we assume that $\mathscr{V}_{\uparrow \infty} \cap \mathscr{V}_{-w} \neq \emptyset$. Evidently we get also $\mathscr{V}_{<\infty} \cap \mathscr{V}_{-w} \neq \emptyset$ . So we can take $v \in \mathscr{V}_{\uparrow w} \cap \mathscr{V}_{-\infty}$ and $v^{\prime} \in \mathscr{V}_{<\infty} \cap \mathscr{V}_{-w}$ such that $\left(v, v^{\prime}\right) \in \mathscr{E}$ holds.

Then we find that hop values on $v$ is larger than $h$. That is, $t \geq f(h+1)$ implies $H_{\varphi, \rho}(t, v, w)>h$.

Now, we take arbitrary $t \geq f(h \dot{+} 1)$. If we take $t$ large enough, condition (2) derives that

$$
\begin{aligned}
h & <H_{\varphi, \rho}(t+1, v, w) \\
& =\rho\left(t^{\prime}, H_{\varphi, \rho}(t, v, w), W\right) \\
& =\min W \\
& \leq H_{\varphi, \rho}(t, x, w)+\varphi(t,(v, x))
\end{aligned}
$$

for each $x \in N(v)$.

Fortiori that holds for $x=v^{\prime}$. That contradicts the selection of $h$.

Now we get $\mathscr{V}_{\uparrow \infty} \cap \mathscr{V}_{-w}=\emptyset$, that is, $\mathscr{V}_{\uparrow \infty} \subset \mathscr{V}_{-w}$ holds.

Lemma 2.9. The routing protocols specified in Example 2.7 and Definition 2.10 both satisfies the assumption of Theorem 2.8 and condition (2).

Proof. Easy to check.

COROLLARY 2.10. We fix the destination $w \in \mathcal{V}$ for the protocols specified in Example 2.7 ans Definition 2.10 . 
Then the convergency of the delay function on $v$ is equivalent to $v \in C_{\varphi, \infty}(w)$ for any vertices $v \in \mathscr{V}$ :

Proof. This corollary follows from Theorem 2.6, Lemma 2.7, Theorem 2.8 and Lemma 2.9 .

\section{The convergency of typical routing protocols}

\subsection{The RIP}

TheOREM 3.1. Let $\left\{T=\omega, V=F_{n}, G=\{\mathscr{V}, \mathscr{E}\}, C=\mathscr{C}_{G}^{\mathrm{RIP}}, \rho=\rho_{\min }\right\}$ be RIP. If we are given a convergent crowdness function $\varphi$, we obtain $H_{\varphi, \rho}(\cdot, v, w)$ is a constant for each $v, w \in \mathcal{V}$ after some finite times.

Proof. This theorem follows to Corollary 2.5 immediately.

$$
u \quad v \quad w
$$

FIGURE 3. The sub graph consisted of three vertices.

\subsection{The hello routing protocol}

Theorem 3.2. Let $\left\{T=\omega, V=\omega+1, G=\{\mathscr{V}, \mathscr{E}\}, C=\mathscr{C}_{G}, \rho=\rho_{\min }\right\}$ be a Hello routing protocol. If $G$ has a connected component which contains more than or exactly three vertices, there exists some convergent crowdness function $\varphi$ such that $H_{\varphi, \rho}(\cdot, v, w)$ divergents for some $v, w \in \mathcal{V}$.

Proof. $G$ has a sub graph which contains just three vertices as shown by Figure 3 .

We define a crowd function $\varphi$ as follows,

$$
\begin{aligned}
& \varphi(t,(u, v))=1, \\
& \varphi(t,(v, w))= \begin{cases}1, & 0 \leq t \leq 2, \\
\infty, & 3 \leq t,\end{cases}
\end{aligned}
$$

then we are able to easily calculate:

$$
\begin{aligned}
& H_{\varphi, \rho}(t, v, w)= \begin{cases}\infty, & t=0, \\
\lfloor(t+1) / 2\rfloor, & t \geq 1,\end{cases} \\
& H_{\varphi, \rho}(t, u, w)= \begin{cases}\infty, & t=0,1, \\
\lfloor t / 2\rfloor+1, & t \geq 2 .\end{cases}
\end{aligned}
$$


We write down the first some times to Table 1.

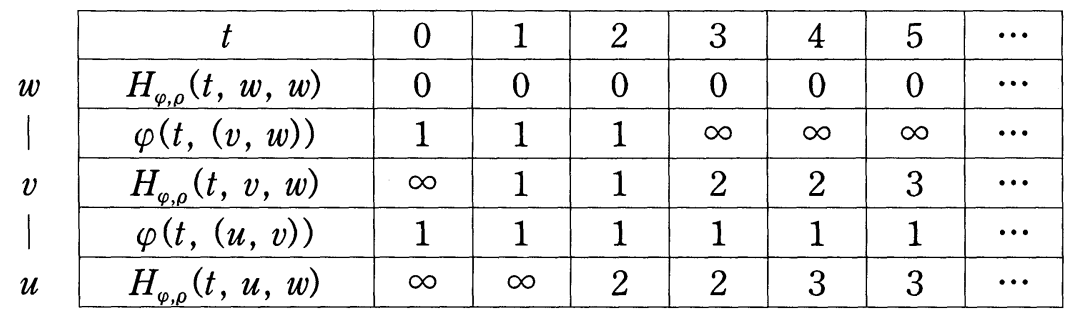

TABLE 1. An example of devergence.

If $G$ has more than three vertices, we can take crowdness function's value $\infty$ on edges except Figure 3.

\subsection{Decreasing routing protocol}

THEOREM 3.3. Let $\{T=\omega, V=\omega+1, G=\{\mathscr{V}, \mathscr{E}\}$ be the decreasing routing protocol where $\left.C=\mathscr{C}_{G / t_{p}}, \rho=\rho_{\downarrow}\right\}$, and we assume that $t_{p}>\# \mathscr{V}$.

Then for given convergent crowdness function $\varphi$ and each $v, w \in \mathscr{V}, H_{\varphi, \rho}(\cdot, v, w)$ terminates after some finite time.

Proof. Corollary 2.4 says that $H_{\varphi, \rho}(\cdot, v, w)$ converges to a finite value or divergent including converges to $\infty$.

On the other hand, Corollary 2.10 says that it converges to a finite value if and only if $v \in C_{\varphi, \infty}(w)$.

In order to prove the theorem, we show that $H_{\varphi, \rho}(\cdot, v, w)$ converges to $\infty$ for each $v \notin C_{\varphi, \infty}(w)$.

Put $m(t)=\min _{v \notin C_{\varphi, \infty}(w)}\left\{H_{\varphi, \rho}(t, v, w)\right\}$. The Definition 2.10 of the routing says that $m(t)$ is a increasing function.

Let $t_{0}$ be a suitable multiple of $t_{p}$, and consider $t$ such that $t_{0}<t \leq t_{0}+t_{p}$.

Definition 2.10 also says that there exists $h_{v, w}(<\infty) \in V$ and $\left(t_{0} \leq\right)$ $t_{v, w}\left(\leq t_{0}+t_{p}\right) \in T$ such that

$$
H_{\varphi, \rho}(t, v, w)= \begin{cases}h_{v, w}(<\infty), & t_{0}<t \leq t_{v, w}, \\ \infty, & t_{1}<t \leq t_{0}+t_{p} .\end{cases}
$$

Then we find $H_{\varphi, \rho}(t, v, w)=\infty$ for the vertex $v$ that satisfies $m(t \dot{-} 1)=$ $H_{\varphi, \rho}(t-1, v, w)$ for each time $t$.

It leads that at least one vertex arrives at $\infty$ for each time $t$ step.

If we consider the last time $t=t_{0}+t_{p}$, we find every vertices $v \notin C_{\varphi, \infty}(w)$ arrive at $\infty$, that is $H_{\varphi, \rho}(t, v, w)=\infty$. 
The increasing function $m(t)_{t \in T}$ arrives at $\infty$, that is, it converges to $\infty$. So we get that $H_{\varphi, \rho}(\cdot, v, w)$ converges $\infty$ for every vertices $v \notin C_{\varphi, \infty}(w)$ also.

\section{REFERENCES}

[1] Bellman, R. E., Dynamic programming, Princeton University Press, Princeton, N. J. (1957).

[2] Bertsekas, D. P., and Gallaher, R. G., Data Networks, Prentice-Hall, Englewood Cliffs, N. J. (1987).

[ 3 ] Comer, D. E., Interworking with TCP/IP, Prentice Hall, Englewood Cliffs, N. J. (1991).

[4] Ford, L. R. Jr., and Fulkerson, D. R., Flows in Networks, Princeton University Press, Princeton, N. J. (1962).

[5] Hedrick, C., Routing information protocol, Request for Comments, 1058, DDN Network Information Center, SRI International (June 1988).

[6] Jech, T., Set Theory, New York, Academic Press (1978).

[7] Kenneth, K., Set Theory. An Introduction to Independence Proofs, Amsterdam, Northholland (1980).

[ 8 ] Malkin, G., RIP Version 2 protocol analysis, Request for Comments, 1387, DDN Network Information Center, SRI International (January 1993).

[9] Malkin, G., RIP Version 2 carrying additional information, Request for Comments, 1388, DDN Network Information Center, SRI International (January 1993).

[10] Mills, D. L., DCN local-network protocols, Request for Comments 891, DDN Network Information Center, SRI International (December 1983).

[11] Mills, D., and Braun, H-W., The NFSNET Backbone Network, Proceedings of ACM SIGCOMM '87 (August 1987).

[12] Moy, J. (Editor), OSPF protocol analysis, Request for Comments, 1245, DDN Network Information Center, SRI International (July 1991).

[13] Moy, J. (Editor), Experience with the OSPF protocol, Request for Comments, 1246, DDN Network Information Center, SRI International (July 1991).

[14] Moy, J., OSPF Version 2, Request for Comments, 1247, DDN Network Information Center, SRI International (July 1991).

Matsushita Information Systems

Research Laboratory Nagoya Co., LTD.

Shirakawa Building Annex

2-6-1 Sakae Naka-ku, Nagoya 460

Japan

E-mail address: kawamoto@miln.mei.co.jp 